\title{
De la ciudad "mestiza" al campo "indígena": internados indígenas en el México posrevolucionario y en Bolivia ${ }^{1}$
}

\author{
From Mestizo City to Indian Countryside: \\ Indian Boarding Schools in Postrevolutionary \\ Mexico and Bolivia
}

\section{Laura Giraudo}

Escuela de Estudios Hispano-Americanos, CSIC, Sevilla

El artículo estudia los internados indígenas en el México posrevolucionario y en Bolivia, considerando las similitudes en sus desplazamientos desde la ciudad al campo y las formas específicas en que cada elite nacional discutía acerca de la educación indígena. Examina también las relaciones entre educadores mexicanos y bolivianos, y sus visiones de la experiencia del otro país. El análisis sugiere que estas experiencias y debates resultan reveladores de ideas comunes acerca de la ciudad (moderna y civilizada) frente al campo (primitivo y atrasado), así como de la idea del campo como el lugar natural y apropiado para el indígena.

PALABRAS ClaVE: Internados; Educación indígena; Indigenismo; México; Bolivia.

This article examines Indian boarding schools in postrevolutionary Mexico and Bolivia, considering similarities in their removal from city to countryside, but also the very specific way each national elite discussed Indian education. It also focuses on relationships between Mexican and Bolivian educators, and their views on the other country's experience in the field. As the analysis suggests, these experiences and debates are quite revealing of widespread ideas about the city (modern and civilized) and the countryside (primitive and backward), and about the countryside as the Indian natural proper place.

KEYwORDS: Boarding Schools; Indian Education; Indigenismo; Mexico; Bolivia.

1 Este trabajo forma parte del proyecto de investigación "El indigenismo interamericano: instituciones, redes y proyectos para un continente, 1940-1960" (HAR2008-03099/HIST) financiado por el Ministerio de Ciencia e Innovación de España. 
La importancia de los proyectos educativos y culturales en el México posrevolucionario ha sido reconocida y analizada de forma creciente por los historiadores tanto en relación a los aspectos inherentes a la educación y a la cultura, como por sus implicaciones en la construcción del Estado y de la nación entre los años veinte y cuarenta. ${ }^{2}$ En el discurso oficial y en los proyectos que se implementaron, la llamada "cuestión indígena" ocupaba un lugar privilegiado. En muchos aspectos, más allá de las peculiaridades del caso mexicano, la cuestión indígena y la ambición de transformar a la población indígena y campesina eran parte de los proyectos y de las realidades de otros países latinoamericanos, que en esa misma época consideraban a la educación/aculturación el mejor instrumento para la modernización y "civilización" de sus sociedades (especialmente su componente indígena) en el marco de la redefinición de su proyecto de nación. El mismo debate se desarrollaba, de hecho, en el ámbito continental (incluso internacional), gracias a continuos intercambios de ideas y a la atenta mirada sobre las experiencias de los demás países.

Este artículo se ocupa del tema específico de los internados indígenas en México y en Bolivia, analizando algunos casos emblemáticos: la Casa del Estudiante Indígena (1926) y los Centros de Educación Indígena (desde 1932) en México y, para el caso boliviano, las escuelas normales para indígenas de La Paz (1911 y 1931) y la escuela-ayllu de Warisata (1931). Las experiencias de los internados son parte importante del debate sobre el concepto mismo de "educación indígena", en el sentido de educación "especial", y, además, resultan reveladoras de la percepción y de la imagen que las elites de estos países tenían de la ciudad (moderna y civilizada) frente al campo (primitivo y atrasado), junto con la idea del campo como el "medio natural" de los indígenas. El recorrido es aparentemente el mismo en los dos casos: los internados se trasladaron desde la ciudad hacia las regiones consideradas más indígenas. Nos proponemos profundizar este paralelismo entre México y Bolivia, revelando como este recorrido, más que representar un simple desplazamiento territorial o cronológico, es expresión de posiciones distintas acerca de la forma en la cual llevar a cabo la educación de estas poblaciones y, en consecuencia, de la forma en que se transforma el proyecto de nación en los dos países.

2 Vaughan, 1999 y 2000; Dawson, 2004; Lewis, 2005; Vaughan y Lewis, 2006; Giraudo, 2008; Rockwell, 2007. 
Además, todo ello conlleva implicaciones en relación a la manera en que el recuerdo y la memoria de estas experiencias han determinado interpretaciones y lugares comunes acerca de la educación indígena en el periodo posterior, con huellas persistentes incluso en la misma historiografía.

Tras el análisis del caso mexicano y del boliviano, nos acercaremos a las relaciones y a los intercambios de ideas entre los dos países, considerando la visita efectuada por el mexicano Adolfo Velasco a la escuelaayllu de Warisata en 1939, y las opiniones de los educadores bolivianos, en particular Elizardo Pérez, Carlos Salazar y Rafael Reyeros, sobre la experiencia mexicana. Finalmente, concluiremos con el Primer Congreso Indigenista Interamericano (Páztcuaro, México, 1940), en el cual los delegados mexicanos y bolivianos fueron los protagonistas del debate sobre el tema de la educación indígena y las experiencias de estos dos países, junto con la de Estados Unidos, el modelo de referencia para las resoluciones adoptadas.

\section{Los indios en la ciudad y los indios en "su medio": de la Casa del Estudiante Indígena a los Centros de Educación Indígena (México)}

de lo que se trataba era de hacer un experimento psicológico
en que el material había de ser indios puros

(SEP, 1927, p. 46)

cazando muchachos aborígenes en edad escolar para llevárselos maniatados a Mezquitic, donde corría fama de que los engordaban para comérselos

(Rubín, 1954, p. 79)

Anunciada por primera vez en junio de 1924 por Manuel Puig Casauranc, futuro secretario de Educación, la Casa del Estudiante Indígena empezó sus actividades en la Ciudad de México en enero de 1926. Su dirección recayó en el profesor Enrique Corona Morfín, que había dirigido el Departamento de Cultura Indígena de la Secretaría de Educación Pública (SEP) y se encargaría más tarde de las misiones culturales. La Casa y sus alumnos fueron utilizados simbólica y políticamente, ya sea por sus partidarios como por sus opositores, y la experiencia - a pesar, o quizás gra- 
cias, a su declarado fracaso- dejó huellas profundas en el debate y en la política educativa posterior. ${ }^{3}$

El proyecto preveía la formación de jóvenes representantes de diferentes grupos indígenas del país que, al terminar sus estudios, tenían que volver a sus comunidades de origen con el objeto de "integrarlas en la cultura nacional". Mediante el cambio de mentalidad y de costumbres de los alumnos, la SEP buscaba formar "difusores de cultura" que desempeñarían el papel de intermediarios con las comunidades rurales. Además se buscaba demostrar las capacidades intelectuales de los indígenas, sometiéndolos a exámenes físicos y mentales para probar que, tal como los mestizos, eran capaces de asimilar la cultura y la civilización dominantes.

Desde sus inicios, el experimento produjo distintas y en ocasiones contradictorias posiciones acerca de la "raza" y la asimilación. Las dificultades surgieron de inmediato, cuando se trató de escoger a los alumnos. Un primer grupo no fue aceptado, por considerarse que estaba formado por mestizos y no por "indios puros". La segunda selección reunió a 198 indígenas que llegaron finalmente a la Ciudad de México. De éstos, se declaró que 180 eran indígenas y sólo 18 mestizos. Curiosamente, muchos de los que se consideraron mestizos eran bilingües, mientras que entre los "indios puros" había varios que únicamente hablaban castellano. ${ }^{4} \mathrm{~A}$ estos últimos se les enseñó la lengua indígena de sus regiones de procedencia, una decisión sorprendente y contrapuesta a la política lingüística oficial de castellanización directa, pero justificada con el hecho de que, al volver a sus comunidades nativas, ese conocimiento les sería indispensable en su labor de promotores sociales y culturales.

Los alumnos eran en su mayoría "presuntos indios puros", varones, de entre catorce y dieciocho años de edad, procedentes de regiones indígenas y residentes en pequeñas comunidades, con primer o segundo año de educación primaria, y dotados de inteligencia, vigor físico y salud. Al llegar a la Casa, cada alumno tenía que contestar una serie de preguntas relativas a sus antecedentes personales, familiares y a la región de origen. Estos cuestionarios nos hablan de las ideas acerca de los grupos indígenas que prevalecían en la SEP, más que de los jóvenes. ${ }^{5}$ El haber reunido a un grupo de

3 Por ello, se convirtió en referencia imprescindible en los estudios sobre educación y, sobre todo en los últimos años, ha sido también objeto de estudios específicos. Véase, entre otros: Aguirre Beltrán, 1973, pp. 126-134; Loyo 1996a; Loyo, 1999, pp. 292-301; Dawson, 1998; Dawson, 2001; Dawson, 2004, pp. 22-29; Giraudo, 2008, pp. 104-125 y 231-278.

4 SEP, 1927, p. 47: Giraudo, 2008, pp. 107-111.

5 SEP, 1927, pp. 35-41. 
distinta procedencia (27 "razas indígenas" según la información oficial) ofrecía la ocasión de averiguar - por medio de exámenes y tomas de medidas realizadas por dos médicos del Departamento de Psicopedagogía e Higiene- cuáles podían ser los criterios psicofísicos para diferenciar a los indígenas entre sí y del resto de la población. La definición de "indios puros" era uno de los retos del experimento pero sus criterios nunca fueron establecidos claramente: si bien estaban influidos por el determinismo racial, los empleados de la Casa y su director optaron, en la mayor parte de los casos, por explicaciones culturalistas para regresar al determinismo biológico en alguna ocasión. ${ }^{6}$ Algunos profesores, como Antonio Gutiérrez y Oliveros, tomando como ejemplo a los alumnos, llegaron a hablar de una superioridad racial de los indios. ${ }^{7}$ En términos generales, a pesar de sus prácticas de fijación racial, la experiencia contribuyó a dar credibilidad a la interpretación de tipo cultural.

Por otra parte, el problema principal fue que la llamada incorporación resultó tan eficaz que la primera generación de alumnos no quiso volver a sus comunidades. Como solución, a pesar de su ubicación en la capital, en 1928 se transformó la Casa en una escuela normal rural. ${ }^{8}$ El nuevo objetivo, la formación de maestros, debía garantizar el retorno de éstos a las comunidades indígenas y justificaba aún más el uso de las lenguas nativas, instrumento que, junto a la "sangre india", ayudaría a los egresados a ganarse la confianza de sus hermanos de raza. ${ }^{9}$ La ubicación urbana implicó problemas organizativos, como por ejemplo la dificultad de realizar las prácticas agrícolas consideradas indispensables en la capacitación de un maestro rural. ${ }^{10}$

Desde octubre de 1930, Rafael Ramírez, encargado del Departamento de Escuelas Rurales, planteará sus dudas acerca del proyecto. Ramírez con-

6 En 1931, por ejemplo, el director expulsó a un grupo de estudiantes "agitadores", que de repente fueron "descubiertos" como mestizos. Acerca de los criterios de definición en la Casa y en general en la SEP, véase Dawson, 2001, pp. 336-343; Dawson, 2004, pp. 22-24; Giraudo, 2008, pp. 99-116.

7 Gutiérrez y Oliveros, 1929.

8 Archivo Histórico de la Secretaría de Educación Pública (AHSEP), Dirección de Misiones Culturales (DMC), Escuelas Normales Rurales (ENR), Casa del Estudiante Indígena, 41, 8, fs. 8-18, "Informe de las labores desarrolladas en el Dpto. Normal Rural inaugurado el 1 de febrero de 1928 en esta Casa”, 21 de junio de 1928.

9 "Informe del Departamento de Escuelas Rurales e Incorporación Cultural Indígena", SEP, 1931, p. 16.

10 AHSEP, Departamento de Escuelas Rurales (DER), 6214, 13, f. 31, "Informe que el director de la Casa rinde a la Superioridad, en relación con las actividades desarrolladas por el Plantel, durante el año de 1929”, 31 de diciembre de 1929. 
sideraba antieconómica la incorporación de individuos (método de la Casa) en vez de grupos (método de las escuelas rurales en regiones indígenas). Sugería reemplazar la escuela por cuatro o cinco internados en las regiones indígenas, una propuesta que se llevó a la práctica poco después. ${ }^{11}$ En respuesta a las críticas, el director Corona enumeraba los logros conseguidos y afirmaba que la Casa había "anulado la distancia evolutiva que separaba a sus indios alumnos de la época actual, transformando su mentalidad, tendencia y costumbres, incorporándolos plenamente a la comunidad social mexicana y sumándolos a la vida moderna". ${ }^{12}$

La Casa del Estudiante Indígena fue finalmente clausurada tras una inspección especial encargada por Ramírez a Manuel Mesa Andraca. Según Corona, se trató en realidad de una acuciosa "inquisición" que celaba desde el principio la intención de liquidar la experiencia. ${ }^{13}$ El informe de Mesa Andraca consideraba que el plantel no había logrado sus objetivos, ya que sus alumnos no habían vuelto a sus comunidades y como escuela normal no tenía buenos resultados, además de resultar excesivamente costosa. ${ }^{14} \mathrm{En}$ realidad, sabemos que los egresados trabajaron desde 1930 en varias comunidades rurales, indígenas y mestizas, y que en varios casos fue la organización misma de la SEP la que les impidió el desempeño de su misión. ${ }^{15}$

En los primeros años treinta, la frustración y decepción por las dificultades que encontraba la escuela rural en el desarrollo de las múltiples tareas que se le habían encomendado condujo a los educadores y funcionarios de la SEP a rediscutir los métodos empleados hasta entonces y a la reforma de varias instituciones rurales. En cuanto a los internados, la SEP, en lugar de traer los indígenas a la "civilización", optó, como ya había sugerido Ramírez, por llevar la "civilización" a los indígenas, estableciéndolos en las zonas rurales: los Centros de Educación Indígena. ${ }^{16}$

11 Ibidem, 36, fs. 1-2, Rafael Ramírez al subsecretario de Educación, 27 de octubre de 1930.

12 AHSEP, DER, Estado de México, 1327, 1400/10, "Informe que el director de la Casa del Estudiante Indígena rinde al Departamento de Escuelas Rurales, Primarias Foráneas e Incorporación Cultural Indígena, acerca de las labores desarrolladas en el propio plantel durante el año de 1930", diciembre de 1930. Cita en f. 10.

13 Ibidem, 849, 1474/41, fs. 11-13, "Informe del Director de la Casa Enrique Corona desde enero hasta mayo de 1932", 31 de mayo de 1932.

14 Manuel Mesa Andraca, "Informe del Visitador Especial sobre la Casa del Estudiante Indígena”, en SEP, 1932, Tomo I, pp. 25-80.

15 Giraudo, 2008, pp. 231-278. Sobre el caso del primer ex alumno de la Casa que trabajó en el sistema de educación federal, Giraudo 2007.

16 Todas las escuelas normales rurales, desde 1926 hasta 1943, estaban organizadas como internados mixtos y en muchas de ellas los alumnos eran de procedencia indígena. Aquí nos ocupamos sólo de los internados indígenas, ya que nos interesa la defensa de esta institución específica. 
Erigidos en el "corazón” de las regiones indígenas, estos internados iban a educar a los alumnos sin "desarraigarlos de su medio": debían diseñar programas únicos, fundados en las condiciones locales, la cultura y el lenguaje de la comunidad en que se situarían, promoviendo además la participación de los vecinos. ${ }^{17}$ A diferencia de la Casa, además de varones, podían ingresar también mujeres. Al igual que en la Casa, los alumnos debían ser de "pura raza indígena" y usar habitualmente un idioma nativo. El plan de estudios incluía la enseñanza básica del español hablado y escrito, de nuevos hábitos y costumbres, el estudio de las artes populares de la región, enseñanzas técnicas, de labores agrícolas y deporte. Se enfatizaba el aprendizaje de las tecnologías modernas en la agricultura, consideradas necesarias para elevar el nivel de vida: a tal fin cada internado debía disponer de 25 hectáreas de tierra para su cultivo. ${ }^{18}$

El objetivo final seguía siendo el mismo, que los alumnos emergieran al final como líderes y "agentes del progreso", uniendo las mejores calidades de sus culturas con los valores educativos. Ahora, sin embargo, los alumnos tenían que mantenerse relacionados con estas culturas y su entorno. $\mathrm{Su}$ especificidad, con respecto a otras instituciones establecidas en esos mismos años, residía en la idea de que debían estar abiertos a la influencia mutua entre comunidad y maestro y ser flexibles para adaptarse al medio. El énfasis acerca de las culturas locales significaba para algunos una educación más rudimentaria (considerando las "limitadas capacidades" de los alumnos) pero, al mismo tiempo, creaba la posibilidad de que las comunidades tuvieran un papel en la definición del contenido de la enseñanza. Estos centros se podían convertir así en lugares donde los indígenas reclamaran tanto el derecho a la educación como el derecho a mantener su propia cultura. ${ }^{19}$

Tras establecer dos internados piloto, el de San Gabrielito en Guerrero y el de Yoquivo en Chihuahua, en 1933 la SEP inauguró otros nueve Centros. ${ }^{20}$ Algunos de los egresados de la Casa, al ser maestros bilingües, se ubicaron en los internados. Desde el primer momento, sin embargo, hubo problemas de organización, de instalaciones, materiales, de falta de

17 SEP, 1931, pp. 11-13; Informe de Rafael Ramírez, jefe del Departamento de Enseñanza Rural y Primaria Foránea, en SEP, 1933, Tomo I, p. 12 y tomo II, pp. 18-23.

18 Véase: Loyo, 1996b; Dawson, 2004, pp. 34-66; Greaves, 2006.

19 Dawson, 2004, pp. 36-37.

20 Informe de Rafael Ramírez, jefe del Departamento de Enseñanza Rural y Primaria Foránea, en SEP, 1933, Tomo I, p. 12 y tomo II, pp. 18-23. 
experiencia del personal, etc., con el resultado de que, después de sólo un año de actividad, muchos internados se habían quedado casi vacíos. El inspector Luis Monzón se encargó de una inspección especial entre 1933 y 1934 y propuso una reorganización fundada en el principio de que "los indígenas sólo podían ser redimidos por los indígenas mismos". ${ }^{21}$

En 1936, gracias al papel que se le asignó en el proyecto cardenista, su número creció hasta 32, involucrando unos 3000 estudiantes. ${ }^{22}$ El contexto favorable ocasionó un gran número de solicitudes desde las comunidades para la apertura de nuevos internados: en ellas se percibe un uso estratégico de la pertenencia étnica —y del "atraso" que a ella se asociaba- para excluir a los no indígenas de estas escuelas. La idea de que los internados debían adaptarse a las especificidades locales abría la posibilidad de pedir privilegios especiales por ser indígenas. ${ }^{23}$

En el plan de trabajo de los nuevos internados, Carlos Basauri, jefe del recién creado Departamento de Educación Indígena, proclamaba el abandono de la búsqueda de los "indios puros" y la equivalencia entre raza y cultura: era suficiente que una comunidad o un individuo correspondieran al tipo de "cultura indígena" para considerarlos dentro de su radio de acción. A pesar de ello, el mismo Basauri afirmaba, pocas líneas después, que "la edad mental de los indios discrepa notablemente de la edad cronológica, siendo su cociente intelectual muy bajo". ${ }^{24}$ Algo que parecía haberse resuelto años antes con los resultados de la Casa - gracias a la demostración de las capacidades intelectuales de sus alumnos- seguía siendo parte del discurso de la SEP.

Los Centros debían ahora organizarse como "escuelas de trabajo" y como un modelo de "comunidad en pequeño, donde los alumnos actúen como miembros de una colectividad de tendencias socialistas: autogobierno escolar, formación de cooperativas de producción, de consumo o mixtas". ${ }^{25}$

21 Dawson, 2004, pp. 38-41.

22 Véase el discurso de Lázaro Cárdenas al abrir el Congreso las sesiones ordinarias en 1937 y en 1940, en SEP, 1976, p. 235 y p. 242. Para la distribución de los centros por Estados, cfr. "Plan general de trabajos de los Centros de Educación Indígena para el año de 1937”, en SEP 1937, pp. 406407.

23 Para casos de solicitudes desde las comunidades, Dawson, 2004, pp. 44-51. También Vaughan, 2000, pp. 274-278 acerca de los internados yaqui. Este mismo proceso de apropiación estratégica del discurso de la SEP ha sido también notado en relación a las escuelas rurales, tanto en las solicitudes de aperturas como en las protestas en casos de clausura, véase Giraudo, 2008, pp. 221-229.

24 "Plan general de trabajos de los Centros de Educación Indígena para el año de 1937", en SEP, 1937, pp. 401-407. Cita en p. 403.

25 Ibidem, p. 404. 
En enero de 1938, la educación indígena pasó a depender del Departamento de Asuntos Indígenas, creado en 1936. Sin embargo, con la administración avilacamachista, se fueron multiplicando las posturas contrarias a la existencia de instituciones educativas especiales. En el marco de un proyecto tendiente a la unificación de la educación (que llegó a eliminar las diferencias entre escuelas urbanas y rurales), el secretario Octavio Véjar Vásquez rechazó la existencia de lugares que "segregaran" a los grupos indígenas. En 1942 los centros de educación fueron transformados en Centros de Capacitación Económica y su número reducido a diecinueve. Su programa de trabajo era de carácter esencialmente práctico, teniendo como actividades principales las labores agropecuarias, la enseñanza de industrias regionales y sólo "instrumentos básicos de la cultura". Se mantenía el uso del idioma nativo y se exigía a los egresados que volvieran a su comunidad de origen. Muchos de los cambios que experimentaron los internados en los años cuarenta fueron más nominales que reales, pero hubo otros cambios que reflejaron la nueva época: el campo dejó de ser tarea prioritaria, dentro de un nuevo proyecto económico fundado en la industrialización. La tendencia a reubicar los centros en zonas semiurbanas o urbanas implicó que disminuyera la importancia de la enseñanza agrícola. ${ }^{26}$

Los éxitos y fracasos de los internados están en parte relacionados con circunstancias y contextos específicos, que no admiten generalizaciones indebidas. Lo que sí podemos afirmar es que se generalizó en México, en el discurso educativo/indigenista posterior (e incluso en la historiografía), una imagen acerca de los Centros de Educación Indígena — así como de la Casa del Estudiante Indígena- en la que se enfatizaron sus fracasos y la imposibilidad de cumplir con sus objetivos como emblemáticos de los fracasos del indigenismo posrevolucionario. En palabras de Aguirre Beltrán,

En uno de estos centros [de capacitación económica] — que concentra a indígenas procedentes de los más diversos grupos étnicos- se pretende impartir enseñanza profesional para enfermeras, educadoras y trabajadoras sociales. Se repite — esta vez con mujeres y con idénticos resultados - el fallido experimento de la Casa del Estudiante Indígena, apenas diez años después de clausurado el célebre establecimiento y cuando todavía está fresco el recuerdo de sus consecuencias ruinosas. ${ }^{27}$

Llama la atención que el "fracaso" sólo se debatió desde la perspectiva del proyecto posrevolucionario y no hubo ningún debate acerca de las

26 Sobre este punto, véase Greaves, 2006, pp. 104-110 y, para casos, pp. 112-116.

27 Aguirre Beltrán, 1973, p. 145. Véase también pp. 126-134. 
consecuencias de la separación de los niños de sus familias que implicaba su educación en los internados, ni los conflictos y rechazos que esto pudo ocasionar en las comunidades: de ello únicamente encontramos huellas en novelas como La bruma lo vuelve azul de Ramón Rubin.

Por otra parte, la Casa del Estudiante Indígena, primero, y los Centros de Educación Indígena, después, graduaron hombres y mujeres bilingües - en una época en que la política linguiística se fundaba todavía en el dogma de la castellanización directa - que ascendieron en las jerarquías políticas y sociales locales, enfatizando, en lugar de negar, sus pertenencias étnicas. En los años siguientes, los encontramos como maestros, activistas y líderes, antropólogos o interlocutores privilegiados con las instancias gubernativas.

El hecho de que estas experiencias fueran consideradas un fracaso facilitó, en los años cuarenta, la crítica a la idea de una educación especial para los indígenas y el abandono de los internados y de la educación bilingüe. Sin embargo, en los años cincuenta, el recién creado Instituto Nacional Indigenista (INI) volvería a formar "líderes sociales" — ahora llamados promotores culturales bilingües - con el papel de actuar, gracias a su pertenencia étnica, como agentes del Estado introduciendo factores de modernidad, representantes al mismo tiempo del Estado y de la comunidad, en el marco de un complejo juego de negociaciones. ${ }^{28}$

\section{Siempre más lejos de la urbe: de la Escuela Normal de Preceptores de Indígenas a la escuela-ayllu de Warisata (Bolivia)}

Se llega a la conclusión de que las escuelas para indios hay que plantarlas en el propio medio indígena.

Lejos de la urbe

(Reyeros, 1937, p. 136)

Misión de la escuela indigenal es darle [al espíritu del indio] nueva vitalidad, modernizarlo sin abandonar su tradición, civilizarlo sin destruir su vieja cultura ni sus instituciones

(Pérez, 1962, p. 59)

En sus primeros años, la Casa del Estudiante Indígena fue presentada como la institución representativa del compromiso del gobierno posre-

28 Acerca de los “indígenas capacitados”, véase Dawson, 2004, pp. 152-164; Pineda, 1993. 
volucionario con las masas indígenas. ${ }^{29}$ Sin embargo, la idea de que fuera necesaria una educación especial como vía para su incorporación nacional - e incluso la formación a tal fin de un magisterio indígena para los indígenas- no era algo exclusivo de México, sino parte de un debate más amplio presente en varios países latinoamericanos. Ni siquiera fue la Casa un experimento pionero en su género: ya en 1911 se había fundado en La Paz, en el barrio de Sopocachi, la Escuela Normal de Preceptores de Indígenas.

Desde los primeros años del siglo $\mathrm{XX}$, el gobierno boliviano se había preocupado por constituir un sistema educativo nacional y un cuerpo docente profesionalizado, como elementos fundamentales para la integración nacional y la construcción ciudadana. Esto se dio con la influencia de modelos de otros países, y con la participación de personal extranjero. ${ }^{30}$

En el marco de esta aspiración del gobierno liberal, el primer proyecto educativo y "de civilización" — las escuelas ambulantes introducidas en 1905 por el ministro Juan Misael Saracho — se inspiraba en los métodos empleados en Estados Unidos para las campañas de alfabetización hacia indígenas y antiguos esclavos negros. También eran consecuencia del miedo de la elite ante el "peligro indio" y de la convicción de que, una vez educados, los indígenas perderían tal peligrosidad..$^{31}$

A partir de 1910, por el contrario, se empezó a imponer una tendencia hacia una educación diversificada y diferenciada, en la cual se consideraba que las escuelas debían llevar a cabo una formación específica, propia de las distintas categorías de ciudadano. La diferencia educativa se planifica así como adecuación con la diferencia ciudadana: la educación estaba pensada en fuerte relación con una "ciudadanía ramificada y diversificada" ${ }^{32}$ En este contexto podemos situar la idea y la fundación de las escuelas normales indígenas, primero, y de las llamadas "escuelas indigenales", después. Paralelamente, la ambición de formar un cuerpo de maestros nacionales se deslizó hacia la búsqueda de maestros también diferenciados.

29 SEP, 1927, p. 20.

30 Entre 1905 y 1908, la misión pedagógica Bustamante-Guzmán estudió países americanos y europeos y condujo finalmente a la importación de maestros normalistas de Chile, a la creación de un sistema de becas para formación en el extranjero y a la contratación del belga Georges Rouma como director de la primera escuela normal del país, inaugurada en Sucre en 1909.

31 Martinez, 1997 y 1998.

32 Martinez, 2005; Irurozqui, 1999. 
La idea de que los indígenas tenían que recibir una educación especial devino un lugar común en el ambiente intelectual y político. ${ }^{33}$

En 1908, el presidente Montes propuso la creación de dos institutos normales y agrícolas (uno para quechuas y otro para aymaras). Su plan tenía objetivos similares a los que tendrá más tarde la Casa del Estudiante Indígena mexicana:

escoger entre los alumnos indígenas reclutados en diversas zonas del territorio, aquellos que [...] hubieran dado pruebas de inteligencia y superioridad y formar con ellos los cursos normales de maestros fijos y ambulantes de su raza. ${ }^{34}$

En abril de 1911 se fundaba la mencionada Escuela Normal de Preceptores de Indígenas de La Paz en el barrio de Sopocachi, con alumnos procedentes de las regiones altiplánicas. Tenía un doble objetivo: formar a los futuros maestros ambulantes y a los futuros "capataces labradores". Dos años después, la escuela fue trasladada a Guaqui, en las orillas del lago Titicaca. La experiencia no resultó positiva y sólo seis alumnos consiguieron finalmente el diploma. Según algunos observadores, entre ellos el futuro creador de Warisata, Elizardo Pérez, los problemas se debían a que Guaqui era un lugar todavía demasiado urbano y la "escuela del indio" no podía funcionar "fuera de su ambiente natural". ${ }^{35}$ En 1914, fue nuevamente desplazada, esta vez cerca de Patacamaya, en el corazón del altiplano andino, en la provincia "más indígena" del Departamento, donde el gobierno alquiló la hacienda Kullta. La formación duraba tres años tras los cuales los alumnos obtenían el diploma de "mayordomos de campo" (maestros ambulantes). Fue clausurada en 1917. Mientras tanto, otro internado indígena, el de Challapata, también había sido desplazado en 1915 a una hacienda, "El Rosario". Esta búsqueda de la hacienda se podría explicar, de acuerdo a Martinez, por las facilidades que ofrecía al garantizar un terreno, un edificio y una población indígena residente. Su contrapartida

33 La conocida polémica de 1910 entre Felipe Segundo Guzmán y Franz Tamayo definió dos posturas claramente opuestas: la una (Guzmán) según la cual la pedagogía debía mejorar y transformar la raza y la otra (Tamayo) que consideraba que la pedagogía debía adaptarse a la raza. Por otra parte, si el discurso oficial defendía una educación dual (dirigida, por un lado, al sector rural indígena y, por el otro, a un sector urbano indiferenciado), en los hechos se establece un sistema escolar triple y hasta cuádruple dirigido a tres o cuatro "públicos" distintos: los futuros campesinos, las elites urbanas, los sectores urbanos populares y las mujeres. Sobre el cambio de tendencia, véase Martinez, 2010, sobre todo pp. 283-313.

34 Citado en Reyeros, 1937, p. 134.

35 Pérez, 1962, pp. 68-69. 
era que las escuelas participaban del "pongueaje", es decir prestaban servicios gratuitos a los hacendados.

Los progresivos desplazamientos de los internados y sus progresivos acercamientos a lugares siempre "más rurales" y "más indígenas" parecen indicar que se pensaba que la solución estaba en educar al indígena "en" y "para" su medio: "réduire les distances, c'était donc parcourir un chemin de la ville à la champagne pour désindianiser en profondeur en milieu indien" ${ }^{36}$ Como ocurrirá también en México tras la Revolución, se empezó a considerar que el mejor maestro para el indígena era el indígena mismo, y que la ubicación rural de los internados era imprescindible para alcanzar el objetivo de civilizarlos y modernizarlos sin que abandonaran o no quisieran regresar al campo.

En los mismos años se fundaron otras tres escuelas normales: en Umala (1914), Colomi (1916) y Puna (1918). Esta expansión privilegió al mundo andino, que se consideraba el más fácil y rápido de "civilizar", y a los dos grupos étnicos mayoritarios: aymaras y quechuas. Por el contrario, ninguna fue fundada en Santa Cruz. Tampoco estas escuelas, si bien establecidas desde el principio en el ámbito rural e indígena, se salvaron de ser desplazadas, y en diciembre de 1919 la normal de Umala fue trasladada a Pucarani, lugar con mayor densidad de indígenas, mientras que la de Colomi pasó a la propiedad "La Florida". Tras un balance muy negativo (se formaron muy pocos maestros y ninguno fue a trabajar en comunidades), todas fueron clausuradas entre 1921 y $1922 .{ }^{37}$ En 1919, el Estatuto para la Educación de la Raza Indígena reorganizó este sistema educativo, aunque sin efectos, ya que la etapa liberal había llegado a su fin. Algunos de sus principios sí se retomarían en Warisata.

Un decenio más tarde, en 1931, una de las primeras acciones de la recién creada Dirección General de Educación Indigenal fue la fundación de una Escuela Normal Indígena en el barrio Miraflores de La Paz. Volvía así a abrirse en la ciudad una normal indígena. Su director, Alfredo Guillén Pinto, fue a los pocos meses sustituido por el inspector Elizardo Pérez, que criticó su ubicación urbana y el academismo de su enseñanza, considerándola inadecuada para la formación de maestros. Tras renunciar a su cargo, quiso establecer una escuela modelo en un ayllu. El lugar escogido fue Warisata, en donde el mismo año se fundó la Escuela Profesional de

36 Martinez, 2010, p. 322.

37 Ibidem, pp. 316-329; Irurozqui, 1999; Reyeros, 1937, pp. 135-136. 
Indígenas, concebida como un centro experimental para la educación del indio en el altiplano paceño, que debía servir como modelo para otras localidades de la República. ${ }^{38}$

Los trabajos de construcción empezaron el 2 de agosto de 1931:

¡Qué jornadas aquellas! Cientos de indios trabajando sin salario, alegremente, unidos en el 'Ayni' o 'achocall', la fraternal institución del trabajo aymara [...] y todos en conjunto, levantaban los muros del edificio, forma plástica, exterior, de ese otro edificio espiritual que iban construyendo al recuperar la fe en sus destinos y en su condición de grupo social. ${ }^{39}$

De los 150 alumnos iniciales, la escuela llegó a matricular, en 1933, 400 aymaras de distintas edades y de ambos sexos, 150 de ellos como internos. Contaba con cuatro profesores, incluido el mismo director Pérez, un maestro de carpintería, uno de mecánica y otro de albañilería. La escuela disponía de seis hectáreas de tierra, para la producción agrícola y ganadera; funcionaban talleres de tejidos, carpintería, mecánica, cerrajería y tejería.

Las características principales de Warisata, defendidas por Pérez, eran la responsabilidad de los alumnos y el autogobierno: el consejo de administración ("Parlamento Amauta") estaba formado por los padres de familia y el alumnado tenía un sistema de gobierno propio, que regulaba el sistema escolar y el internado. ${ }^{40}$

Precisamente sobre el contexto de su fundación —si Pérez "conquistó" a los indígenas de la región o si fueron ellos los verdaderos promotores de la experiencia - y sobre el grado de participación y protagonismo en la escuela-ayllu se desencadenó una cierta polémica, que ha dejado sus huellas, originando distintas orientaciones historiográficas. ${ }^{41}$

El experimento de Warisata se trasformó en pocos años en una experiencia generalizada por el territorio nacional, con la creación de los llamados "núcleos escolares campesinos". Dentro de éstos se ramificaban pequeñas escuelas unitarias, llamadas "seccionales", que proporcionaban una educación más rudimentaria. Para la coordinación y las directivas se instalaba en un punto céntrico y principal la escuela central o "matriz", de la que

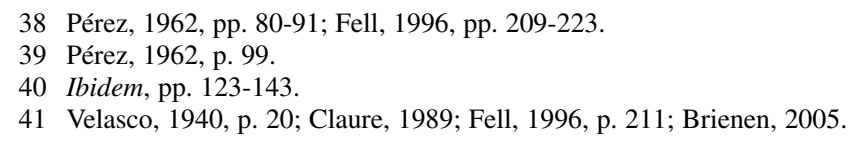


dependían todas las seccionales. ${ }^{42}$ En octubre de 1936 se realizó la primera "Asamblea de Maestros Indigenistas", donde se aprobaron los Estatutos de Educación Indigenal y la Declaración de Principios de la Escuela Campesina. Esta última definía a Warisata como "instituto de Indología y experimentación pedagógica". ${ }^{43}$

En 1937 Elizardo Pérez fue nombrado director general de educación indigenal y campesina. Ese mismo año, en el momento de auge de esta experiencia, el gobierno boliviano decretó el 2 de agosto de cada año como Día del Indio, en homenaje a la fundación de Warisata. El conocido estudioso estadounidense Frank Tannenbaum tenía palabras entusiastas sobre la escuela-ayllu:

Warisata es el jalón más grande por la rehabilitación de los indios [...] Su maravillosa organización, la Escuela Matriz o central rodeada de multitud de pequeñas escuelas que viven en su entorno, en el seno de las comunidades indígenas [...] Esto es muy interesante y no existe en ninguna parte, ni siquiera en México [...] No creo que deban perder el tiempo en polémicas y disertaciones. Mi consejo es que creen en Bolivia veinte Warisatas. ${ }^{44}$

Sin embargo, las actividades de Warisata desencadenaron conflictos con las autoridades locales y los hacendados. La oposición de estos últimos (miembros de la poderosa Sociedad Rural Boliviana) aumentó sobre todo con la creación de una cooperativa de compraventa que se ocupaba de comercializar directamente los productos indígenas en los centros urbanos y de comprar a mejor precio los de primera necesidad..$^{45}$

Las numerosas polémicas que se desataron con respecto a Warisata estaban en parte relacionadas con la acusación a Pérez de una gestión clientelista, pero, sobre todo, el debate era acerca del mismo concepto de "educación indígena", o sea un sistema distinto de las normas vigentes para la

42 En la visión de Pérez, se trataba de revitalizar el sistema cooperativista de la Marka, Pérez, 1962, pp. 188-189. Entre 1936 y 1948, fueron creados un total de 42 núcleos escolares, sumando casi 900 escuelas seccionales. Esta estructura permitió, con escasos recursos, establecer un control sobre las escuelas indígenas que habían surgido sobre todo en los años veinte y que fueron incorporadas a los núcleos. Sobre este punto, Brienen, 2005, pp. 141-146. El núcleo escolar boliviano tiene un parecido con el sistema de los "circuitos rurales", adoptado en México por un breve periodo entre 1928 y 1930, que permitía disminuir notablemente los costos de la expansión del sistema federal de educación. Las escuelas de circuito fueron transformadas en "escuelas de tipo económico", en las cuales los vecinos aportaban parte del sueldo del maestro. SEP, 1929, p. 401 y SEP, 1931, p. 22.

43 Pérez, 1962, pp. 221-227. Cita en p. 225.

44 Ibidem, p. 324

45 Velasco, 1940, p. 20. 
población no-indígena. Empezó así una competencia entre dos tipos de núcleos: el de Warisata y el de Caquiaviri. Este último, dirigido por Alfredo Guillén Pinto (el primer director de la Escuela de Miraflores en 1931), representaba el modelo antiwarisata, con el objetivo explícito de "mestizar" a la población indígena.

Elizardo Pérez, en su obra sobre Warisata, criticaba la experiencia de Caquiaviri:

contra nuestra naciente pero ya vigorosa doctrina, se había inventado otra, elucubrada por normalistas convertidos al indigenismo y por intelectuales deseosos de figuración aunque desprovistos de toda ciencia [...] consistía en oponer a la escuela del ayllu, la escuela de la aldea [...] Caquiaviri es una escuela fundada en la ciudad —equivocadamente — y trasladada luego a la aldea — también equivocadamente-; mientras Warisata revela desde el primer momento una doctrina y visión definida. ${ }^{46}$

En otras palabras, para Pérez el desplazamiento tenía que ser no sólo territorial sino teórico: no sólo de la ciudad al campo, sino de la idea de mestizar a los indios a la idea de revitalizar sus instituciones ancestrales.

A su vez, Rafael Reyeros acusaba a los defensores de las escuelas indigenales de haber malgastado mucho de su esfuerzo en la albañilería: "la pedagogía de la teja y el ladrillo". El énfasis en el trabajo y el desprecio al "intelectualismo", según Reyeros, se aplicaban, igual que en la colonia, "con el sudor del indio". A diferencia de Warisata, "en Caquiaviri, la obra educacional no se estructuró con adobes para formar un edificio". ${ }^{47}$

En evidente polémica, el autor aseguraba que en Caquiaviri los indígenas que trabajaban en forma sistemática recibían un salario, mientras que en Warisata "los indígenas no ponen la mano en las orientaciones pedagógicas de la escuela, sino en su administración, en la cooperación con brazos para las edificaciones". ${ }^{48}$

Reyeros criticaba el sistema de internado y, en general, su actitud es de total desacuerdo con la idea de una "educación especial para indígenas":

Se separa la escuela indígena del resto de las demás [...] se la denomina "educación indigenal", con barbarismo gramatical y todo [...] constituye una rama especial dentro de la educación en general, como si también existiera una educación mestiza y una educación blanca. La educación no admite gradaciones de color de piel, ni se divide por grupos sociales. ${ }^{49}$

\footnotetext{
46 Pérez, 1962, pp. 229-230.

47 Reyeros, 1946, p. 40 y p. II.

48 Ibidem, pp. 156-157.

49 Ibidem, p. 124.
} 
Para fundar su argumentación en contra de la educación especial, mencionaba al mexicano José Vasconcelos y su oposición a que la educación de los indígenas fuera algo especial y separado del resto de la población. ${ }^{50}$

Así llegamos a lo que es el corazón del pensamiento de Reyeros que, de alguna manera, condiciona todo su texto: "Lo capital es bolivianizar al nativo. Homogeneizar al elemento humano". ${ }^{51}$ El objetivo principal de la educación del indígena debía ser acercarlo lo más posible al blanco:

El indio es el germen de un futuro mestizo, como que en cada mestizo queda siempre algo del indio y ya es un blanco en potencia. Porque lo blanco, no es una postura biológica, sino simplemente social. [...] detrás de todo blanco boliviano, asoma el fleco deshilachado del poncho indio y, todo indio, es en sí, un mestizo en potencia y éste, un futuro blanco..$^{52}$

Reyeros no critica, al contrario, la ubicación en el campo de las escuelas indígenas, pero sí su especialidad. Frente a esta posición y en respuesta a la acusación a la escuela de Warisata de ser racista, Carlos Salazar afirmaba que "decir que no existe diferencia alguna entre blancos, mestizos e indios, es justificar la situación de esclavitud de estos últimos". ${ }^{53}$

La declinación de Warisata empezó precisamente cuando su experiencia se había conocido más allá de las fronteras nacionales y recibía muchas visitas de especialistas extranjeros, interesados en ese original experimento de educación indígena. Paradójicamente, mientras que en el Primer Congreso Indigenista Interamericano, celebrado en Pátzcuaro, México, en 1940, se admiraba la experiencia boliviana y se adoptaban resoluciones inspiradas en la escuela de Warisata, en Bolivia se suprimía la Dirección General de Educación Campesina y Elizardo Pérez, que representaba oficialmente a su país en el Congreso, quedaba destituido. ${ }^{54}$

En los años cuarenta, la experiencia de los núcleos escolares no se abandona, pero se moderan los aspectos más radicales de la experiencia de Warisata. ${ }^{55}$ Mientras en otros países andinos (Perú, Ecuador y Guatemala)

50 Ibidem, p. 125 y 158.

51 Ibidem, p. 277.

52 Ibidem, p. 22 у p. 162.

53 Carlos Salazar Mostajo, “iWarisata mia!”, en Pérez, 1962, p. XX. El texto de Salazar fue publicado originalmente en La Calle, La Paz, febrero de 1943.

54 Llama la atención sobre esta paradoja Fell, 1996, pp. 216-127.

55 Las escuelas "indigenales" se reorientaron, enfatizando su papel en la alfabetización, aculturación, y en adaptar las costumbres indígenas a las "más modernas", como se puede deducir de la descripción de Bairon, 1942. 
se adoptan sistemas parecidos, Warisata quedará en Bolivia como una palabra simbólica, y su experiencia y la de las otras escuelas indígenas darán lugar en la historiografía a dos interpretaciones principales, presentadas a veces como incompatibles: símbolos de la resistencia comunitaria y de la estrategia indígena de autopreservación cultural, por un lado, y parte de la estrategia estatal para establecer su control en las áreas rurales y destruir la cultura indígena imponiendo su modernización, por el otro. ${ }^{56}$

\section{Una mirada mexicana sobre Warisata... y miradas bolivianas sobre México}

En 1939, la SEP envió una delegación de maestros mexicanos a Bolivia y uno de ellos, Adolfo Velasco, escribió una monografía acerca de Warisata, publicada en 1940 con ocasión del Primer Congreso Indigenista Interamericano, en el cual fue presentada. ${ }^{57}$ Según Carlos Salazar, "los maestros mexicanos que llegaron ese año, se marcharon a su patria asombrados por la prodigiosa actividad de Warisata". ${ }^{58}$

En su introducción, Velasco afirmaba que quería dar a conocer a todos los que se interesasen por la educación del indio la "institución tipo de Bolivia", con la finalidad de que el ejemplo fuera imitado por todo el magisterio boliviano y por los educadores de cualquier país que en verdad respondieran al papel de maestros y a la conciencia de responsabilidad. Confiaba también en que su trabajo estimulara al gobierno de Bolivia a incrementar las escuelas indigenales. ${ }^{59}$

Tras la descripción geográfica de Warisata y de su entorno, las observaciones acerca de los habitantes del altiplano y de sus condiciones políticas y sociales revelan varios estereotipos referentes a los indígenas que en los mismos años estaban presentes en el discurso indigenista mexicano:

los indios si adolecen de taras espirituales y sociales, también es cierto que disponen de virtudes, de cualidades positivas [...] dichos indios son susceptibles de transformación.

56 Brienen, 2005; Claure, 1989; Choque, Soria et al., 1992.

57 Velasco, 1940, p. I. La parte relativa a Warisata consta de 72 páginas. En el mismo texto, Velasco también incluye un escrito acerca de la Escuela de Recuperación Indígena de Caiza (pp. 7384) y otro acerca de los "indios selvícolas bolivianos" (pp. 85-91).

58 Carlos Salazar Mostajo, “¡Warisata mia!”, en Pérez, 1962, p. XXI.

59 Velasco, 1940, pp. I-IV. 
Con las condiciones apuntadas en que vive el indio, ya se comprende que su psicología es de timidez y además es huraño y medroso. Es analfabeto en su inmensa mayoría y es fanático. ${ }^{60}$

Elizardo Pérez, "el apóstol”, es descrito con estas palabras:

En Bolivia, como en todas las latitudes, siempre surgen hombres que oportunamente intervienen en los hechos trascendentales de la vida de los pueblos, y a esta ley ineludible se debió que entre el magisterio boliviano se perfilara un maestro humilde pero bastante capacitado y con una gran visión del problema indígena, y más que visión, con un sincero amor para la raza esclavizada y una comprensión cabal de la situación social del indio. ${ }^{6}$

Las dificultades encontradas — los escasos recursos, el clima y el entorno, es decir "la oposición de la naturaleza", pero también las amenazas de los latifundistas de la región - son referidas con detalles, ya que, según Velasco, se trata de "un acto de justicia". ${ }^{62} \mathrm{El}$ autor tiene también palabras de admiración para el edificio escolar, levantado con escaso presupuesto y con material del lugar.

Describe de manera detallada tanto las condiciones materiales (edificio, anexos, mobiliario) cuanto la organización y el contenido de la enseñanza impartida (materiales, programas de estudios, distribución de tiempo, clasificación de alumnos). Velasco considera encomiable la decisión de la Dirección de Educación Indigenal de suprimir programas detallados de estudios, dejando libre a cada escuela para formularlos "de acuerdo con las necesidades del medio, la capacidad mental de los alumnos, costumbres y recursos naturales de la región", si bien también se explicitaba en el Reglamento de Educación Indígenal la necesidad de acabar con el regionalismo y construir la idea nacional. ${ }^{63}$

El autor subrayaba el papel de los representantes de las comunidades y de los alumnos en la organización y funcionamiento de las escuelas. La pérdida de tiempo y molestia que estas obligaciones ocasionaban

no es con el fin de explotar al indio, sino que aunque se les hace prestar trabajos personales gratuitos [...] todo ello es con los nobles fines de que contribuya con su esfuerzo y con su sacrificio, a su propia redención y a su propia cultura. ${ }^{64}$

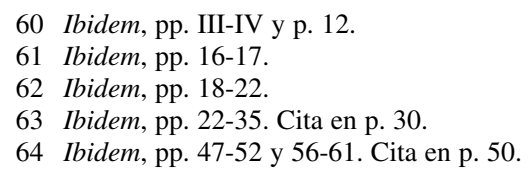


Los primeros frutos de la escuela de Warisata fueron precisamente los jóvenes que estaban adquiriendo el grado de maestros y los alumnos especializados en diversos oficios: "ya capacitados para el desempeño de estas artes, se han derramado en sus propias comunidades". ${ }^{65}$

Velasco interpreta (y defiende) así Warisata con la mirada del educador mexicano que, de acuerdo con la reciente orientación del discurso educativo e indigenista de su país, está observando (y defendiendo) los métodos que permitan alcanzar la transformación del indio por medio de los indios mismos y en su "medio natural".

Curiosamente, a pesar de haber visitado Warisata por encargo de la SEP y a pesar del preámbulo de su obra, Velasco no hacía ninguna referencia a México o a experiencias similares. Al contrario, en la obra de Pérez sobre Warisata se menciona en varios puntos la situación educativa mexicana. Compara las escuelas rudimentarias mexicanas (1911) con las escuelas ambulantes bolivianas y las escuelas rurales (las posrevolucionarias) con las escuelas indigenales. Menciona a Vasconcelos, la creación del Departamento de Educación y Cultura Indígena y la Casa del Estudiante Indígena, equivocándose en su fecha de fundación y en el papel de Vasconcelos: "En 1930 (sic), el mismo Vasconcelos funda en la ciudad de México 'La Casa del Indio', con resultados negativos, y por último se crean los 'Internados Indígenas" ". ${ }^{66}$ Parece presentar a Vasconcelos como un defensor de la educación especial, cuando precisamente el primer secretario de Educación del periodo posrevolucionario se había opuesto a la creación del Departamento de Educación Indígena. Más adelante, Pérez notaba el desfase cronológico entre México y Bolivia:

en 1921, cuando se gestaba en México la escuela que revalorizaría al indio, se cerraban en Bolivia las pocas escuelas normales rurales que habían venido funcionando [...] su clausura correspondía a una definida línea de conducta gubernamental respecto al problema indio. ${ }^{67}$

Unos años antes de la visita de la delegación mexicana a Bolivia, en 1937, el presidente Cárdenas ofreció seis becas para maestros indigenistas bolivianos, con el fin de que pudieran estudiar la organización de la escuela indígena mexicana. El grupo quedó integrado por Rafael Reyeros (jefe

65 Ibidem, pp. 61-62.

66 Pérez, 1962, p. 67.

67 Ibidem, p. 72. 
de la misión), Ernesto Vaca Guzmán, Max Bairon, Toribio Claure, Leónidas Calvimontes y Carlos Salazar. Según Pérez, los dos primeros se habían nombrado aprovechando su ausencia y, de acuerdo a Salazar, entre ellos había un solo maestro indigenista (el mismo Salazar), siendo los demás enemigos de Warisata:

la actuación de la Misión fue desgraciadísima [...] Los adobes de mi escuela, fabricados con sangre, tenían más mérito que los mármoles que pisábamos en los palacios educacionales aztecas. La organización de nuestra escuela era superior a la de cualquier otra institución mexicana. ${ }^{68}$

Reiteraba así la opinión ya expresada en una carta dirigida a Pérez durante la misión, en la que escribía que la obra que se estaba realizando en México era superior por su cantidad, gracias a la gran inversión de recursos, pero no por su calidad. Bolivia era "un país demasiado pobre para poder hacer experiencias": a pesar de los escasos recursos disponibles, la escuela indigenal hacía "obra verdaderamente revolucionaria" y "la obra social de Warisata [era] superior" ${ }^{69}$

Como se imaginará, la opinión del jefe de la misión era otra: Reyeros, al relatar al ministro Peñaranda la visita al internado mexicano de Los Remedios (Ixmiquilpan), enfatizaba lo mucho que quedaba por hacer en Bolivia, reorientando la obra educativa, al igual que en México, a una acción dirigida a toda la comunidad para sus necesidades inminentes (agua, higiene, asistencia médica, etc.). En su respuesta, el ministro reprochaba a Reyeros su desconocimiento del sistema de organización indigenal boliviano, así como del mexicano, y le invitaba a defender "la doctrina boliviana en materia de Educación Campesina" cuyos principios fundamentales eran "trabajo elevado a categoría de elemento esencial de la educación, sentido práctico y utilitario de la enseñanza, arraigo a la industria local, conservación de los sistemas ancestrales de la cooperación en el trabajo" ${ }^{70}$

El mismo Reyeros consideraba que la fundación de la escuela de Sopocachi y, más tarde, la de Miraflores "acusa[ban] un estrecho parentesco con el ensayo mexicano y los lineamientos impresos a la Casa del Estudiante de dicha República", mientras que los internados mexicanos, como el de

68 Salazar en Ibidem, p. XIX.

69 Carta de Carlos Salazar a Elizardo Pérez el 19 de julio de 1938, en Ibidem, pp. 284-288. Citas en p. 287.

70 Reyeros al ministro Peñaranda el 15 de febrero y respuesta de éste último el 17 de marzo de 1938, en Ibidem, pp. 282-284. 
San Gabrielito, podían compararse con la escuela de Warisata, al ser levantados con la cooperación, "voluntaria o impuesta", de los indígenas. ${ }^{71}$

De acuerdo a Pérez, al contrario, eran los Centros de Educación Indígena, a pesar de su ubicación, los que correspondían a los internados bolivianos de Sopocachi o al de Miraflores: "Nada tenía que ver, pues, con nuestro internado, que es una resultante de la organización del Núcleo, integrado a la vida de la marca ancestral". ${ }^{72}$ Es decir, los internados mexicanos habían cambiado su ubicación, desplazándose de la ciudad al campo, pero no tenían todavía una orientación propiamente indigenista, y en esto residía la causa de su fracaso. ${ }^{73}$

\title{
Para concluir: entre México, Bolivia y sus "irradiaciones continentales"
}

\begin{abstract}
Cuando en el Congreso de Páztcuaro los representantes norteamericanos y mexicanos sostuvieron la tesis de que los internados habían fracasado hice ver que fue porque se propusieron sistemas artificiales y exóticos y que en Bolivia se propuso el modelo de la naturaleza.
\end{abstract}

(Pérez, 1962, p. 473)

Tras esta exploración del tema de los internados indígenas entre México y Bolivia, podemos afirmar que el recorrido es sólo aparentemente el mismo: si es cierto que estas escuelas especiales se trasladaron en ambos países desde la ciudad (una ciudad percibida como mestiza/blanca y civilizada) hacia el campo (percibido como indígena y atrasado), el proceso no fue ni lineal -en el caso boliviano, especialmente, hubo idas y vueltas entre ciudad y campo- ni fue interpretado de forma parecida por los educadores mexicanos y bolivianos en su momento, ni ha tenido el mismo papel en las interpretaciones y en la memoria de los dos países. Podemos, desde luego, encontrar parecidos entre las dos experiencias (varios de ellos

71 Reyeros, 1946, p. 154 y 155.

72 Pérez, 1962, p. 283.

73 En otra parte de su obra, dedicada al sistema educativo mexicano, Pérez (pp. 348-349) menciona una encuesta que realizó el internado mexicano de Los Remedios en 1939, preguntando a los alumnos otomíes donde querían vivir y la profesión a la cual querían dedicarse: sus respuestas fueron en la ciudad de México y ser músicos, cuando el objetivo del internado era prepararlos para el campo. Cuando volvió al mismo internado en 1950 obtuvo idénticas respuestas. 
han sido ya mencionados explícitamente en estas páginas), cruzando incluso los internados de la urbe y los de las regiones indígenas: la importancia asignada a los idiomas nativos en la Casa del Estudiante Indígena y en Warisata, por ejemplo, o la defensa de las "calidades del indio" presente en ambas. En otros aspectos, está muy clara la convergencia entre los Centros de Educación Indígena y los núcleos escolares campesinos ${ }^{74}$ y el cambio de tendencia: el énfasis en la "escuela del trabajo" y en la transformación económica; la necesidad de arraigar a los indígenas en su "medio natural" y hacer de ellos agricultores y artesanos modernos. Sin embargo, también hay una clara divergencia en cómo fueron considerados, en el contexto del Primer Congreso Indigenista Interamericano, los resultados de estas escuelas: un fracaso, para unos, y el ejemplo a seguir, para otros.

Celebrado en Páztcuaro (Michoacán, México) en abril de 1940, el Congreso reunió delegados de 19 países americanos. ${ }^{75}$ El Acta Final de Pátzcuaro, con sus 72 resoluciones, representó un acuerdo acerca del significado y los objetivos del indigenismo: allí se defendió la idea de un indigenismo como política especial — fundada en el conocimiento y en el estudio científico - dirigida a un grupo de la población con necesidades particulares, los indígenas. ${ }^{76}$

La sección educativa del Congreso, presidida por el peruano José Ángel Escalante, defendió la enseñanza en idiomas nativos y la idea de que las escuelas para indígenas debían fundarse en "sus regiones". ${ }^{77}$ Las cuatro resoluciones aprobadas en materia educativa reflejaban las experiencias de México, Bolivia y Estados Unidos, y las sugerencias de sus delegados. De ellas, sólo una se presentaba como general, mientras que cada una de las otras tres retomaba, explícitamente, la experiencia de uno de los tres países.

La resolución XXXV — “La política de la educación indígena de la Revolución Mexicana”- proponía la adopción de los siguientes postula-

74 Sin que se desarrolle el tema, este paralelo se sugiere en Loyo, 1996b, p. 144.

75 Los únicos dos países americanos que no enviaron delegados oficiales al Congreso de Pátzcuaro fueron Canadá y Paraguay. Además de las delegaciones gubernativas, participaron invitados especiales, asesores y 47 delegados indígenas procedentes de Estados Unidos, México y Panamá. El resultado más importante del Congreso de Pátzcuaro fue el establecimiento de una institución intergubernamental especializada en la cuestión indígena, el Instituto Indigenista Interamericano (III).

76 "Acta Final del Primer Congreso Indigenista Interamericano. Pátzcuaro, Mich. México", en III, 1940, vol. I, pp. 1-42. Véase también III, 1941.

77 Miembros de la Sección Educativa: presidente José Ángel Escalante (Perú), vicepresidente Williard W. Beatty (Estados Unidos), secretario Julio de la Fuente (México) y relator Gerardo Cabrera Moreno (Colombia). Durante la sesión se presentaron 11 ponencias, por delegados de México, Bolivia, Estados Unidos y Perú. Véase III, 1940, vol. III. 
dos: respeto de la personalidad indígena (esta expresión hacía referencia a "manifestaciones culturales típicas", "hábitos positivos de organización social" y "sentimiento de dignidad personal y colectiva"); reconocimiento de las lenguas nativas y su uso en la enseñanza, junto con la lengua nacional; realización de actividades escolares y extraescolares conformes a su "estado cultural"; aprovechamiento de los elementos esenciales de la vida indígena. Todo ello implicaba la elaboración de textos de enseñanza y la promoción de literatura en lenguas nativas, así como el empleo preferente de "indígenas competentes". La resolución XXXVIII — "Plan de educación indigenal"- tenía en cuenta las propuestas de la delegación boliviana. Recomendaba que la organización de las escuelas se adaptara a las condiciones locales. Debían "levantarse en el corazón mismo de las comunidades indígenas, y sus formas de gobierno [tener] como propósito primordial la elevación de las condiciones sociales y económicas de sus habitantes, con el objeto de no apartarlos de su suelo". Los vecinos y los padres de familia debían tener participación activa en la escuela, de su levantamiento material a sus labores. Se sugería una organización en escuelas centrales (con los grados desde pre-escolar a vocacional) y escuelas elementales en las comunidades. Una escuela especial era reservada a los llamados "silvícolas" y grupos móviles, elaborada gracias a los estudios antropológicos. ${ }^{78}$

Tomando en cuenta lo que conocemos de la experiencia mexicana y de la boliviana, llaman la atención varios aspectos de estas resoluciones, sobre todo sus vacíos. En la resolución "mexicana", no hay, sorprendentemente, ninguna referencia a los internados y, a pesar de la mención que se hace al empleo preferente de "indígenas competentes" para los puestos educativos, no aparece una clara defensa de la importancia de la formación de "maestros indígenas para los indígenas". Es decir, están ausentes los dos aspectos fundamentales de la experiencia mexicana en este campo, la equivalencia entre escuelas indígenas e internados y la importancia asignada a

78 “Acta Final”, en ibidem, vol. I, pp. 19-24. La resolución XXXVI — "Educación indígena”era el resultado de las sugerencias de Estados Unidos y proponía que estas escuelas ofrecieran, tanto a los niños como a los adultos de las comunidades, una serie de servicios; insistía en el empleo de maestros nativos; recomendaba la protección y hasta la promoción de elementos de la cultura nativa. Se recomendaba también que los programas tuvieran en cuenta las necesidades regionales y que fueran "prácticos". Se hacía una sola referencia a la modalidad de los internados, sugiriendo ofrecer "facilidades de residencia" a los alumnos procedentes de zonas remotas. La cuarta resolución sobre educación, la XXXVII, "Experiencias de la escuela rural indígena", constaba de dos puntos: recomendaba la participación de los maestros en las "obras de colonización interior, sin perjudicar los intereses vitales de los indígenas" y la promoción de nuevas actitudes en el proceso educativo, "sin violentar las creencias tradicionales". 
que estos internados fueran también escuelas normales. Cabría sospechar que entre los delegados mexicanos no había acuerdo sobre el camino a tomar y sobre todo acerca de cómo evaluar la experiencia de los internados, sospecha que se confirma al leer las ponencias presentadas. El Departamento de Asuntos Indígenas entregó durante la sesión una serie de disposiciones acerca de los internados, de los cuales se encargaba desde 1938. De ellas hay que destacar el énfasis en la enseñanza práctica, en la formación de "obreros del campo o del taller", pero no se presentó ninguna evaluación de la experiencia. ${ }^{79} \mathrm{El}$ antropólogo Julio de la Fuente presentó una ponencia en la cual relataba algunas situaciones presentes en la Sierra de Oaxaca, y una de sus observaciones se aprovechó para la resolución que llevaba el mismo título, la XXXVII, pero no para la resolución mexicana. ${ }^{80}$ Las referencias al respeto de la "personalidad indígena" y de las lenguas autóctonas de la resolución XXXV derivaban de la ponencia presentada por Luis Álvarez Barret: de hecho, las conclusiones de su ponencia equivalen al texto de la resolución. ${ }^{81}$ En otras sesiones del Congreso se presentaron ponencias de educadores o relacionadas con los temas educativos con opiniones diversas, sin embargo es evidente que los delegados mexicanos no consideraron oportuno presentar una defensa, ni siquiera una reflexión, acerca de la experiencia de los internados indígenas. También en la resolución "estadounidense", la XXXVI, el tema de los internados casi no se menciona, y la única referencia a alumnos residentes se justifica por su procedencia remota, pero no por ser una modalidad educativa apropiada para los indígenas. Sabemos que los delegados estadounidenses tenían posiciones contrapuestas sobre este punto y, si en la sesión educativa se presentó una ponencia bastante benévola hacia la política de internados, en la sesión socio-económica John Collier — junto con Moisés Sáenz, el gran protagonista de Páztcuaro y después del Instituto Indigenista Interamericanodenunció los internados como un camino equivocado y defendió las escuelas diurnas dentro de las reservas. ${ }^{82}$

79 "Principales disposiciones que rigen la labor educativa en las escuelas vocacionales de agricultura para indígenas (antes internados indígenas). Departamento de Asuntos Indígenas”, en ibidem, vol. III, doc. 6.

80 "Experiencias de la escuela rural indígena. Prof. J. De la Fuente", ibidem, vol. III, doc. 4.

81 "La política de la educación indígena en la revolución mexicana. Prof. Luis Álvarez Barret", ibidem, vol. III, doc. 5 .

82 "La educación de los indios en los Estados Unidos. Willard W. Beatty", ibidem, vol. III, doc. 3; "Comentarios sobre el estado, las condiciones presentes y los planes del gobierno de los Estados Unidos de Norteamérica para sus indios. John Collier", ibidem, vol. IV, doc. 10. Sobre esta divergencia, véase Loyo, 2008. 
Por otra parte, la resolución "boliviana", si bien tampoco hacía referencia explícita a los internados, sí recogía los elementos fundamentales de su experiencia y defendía la ubicación ("en el corazón mismo de las comunidades indígenas") y el objetivo ("no apartarlos de su suelo") de las escuelas indigenales. A pesar de lo que escribirá Elizardo Pérez - considerando que Bolivia obtuvo "un gran triunfo en el Congreso [...] logró hacer aprobar los puntos esenciales de su doctrina indigenista" gación boliviana reunía educadores con posiciones contrapuestas. Entre los participantes circuló la obra de Velasco, pero también otro escrito titulado "El Estado de la Educación Indigenal", del Consejo Nacional de Educación, órgano que en los últimos años había manifestado su oposición al modelo de Warisata y a Elizardo Pérez. ${ }^{84}$ Este escrito proclamaba la necesidad de escuelas para indígenas, pero también que éstas no fomentaran las "primitivas" prácticas políticas y culturales del ayllu. En la sesión se presentaron una ponencia y extractos del Reglamento de Educación Campesina aprobado en 1939. En la primera se retomaban argumentos de Pérez y se defendía el núcleo escolar indígena como internado, necesario para "remover las malas costumbres, conservando las buenas", además de su ubicación en el ayllu. El Reglamento, en el que también es evidente la influencia de la experiencia de Warisata, argumentaba la necesidad de una sección del internado para padres y madres, para someterlos a "un tratamiento de vida moderna", ya que "la finalidad de la escuela es preparar el ambiente social a que deben regresar los niños que se transforman en ella" ${ }^{85}$ La resolución resultó bastante más moderada en sus recomendaciones, como era de esperar, debido a los conflictos en el seno de la delegación boliviana.

En ambos países, el contexto político había cambiado y con él las prioridades en la educación de los indígenas: quedaba la idea de arraigarlos en el lugar al que pertenecían "por naturaleza", el campo, y capacitarlos para el trabajo, de acuerdo a las necesidades nacionales de mejoramiento de la producción económica. Sin embargo, el tema de las escuelas especiales, con sus realizaciones, no iba a terminarse con su supuesto fra-

83 Pérez, 1962, p. 340.

84 Ibidem, pp. 121-122 y 333-347.

85 "Procesos y resultados actuales de la campaña de educación indígena en Bolivia. Contribución de la Delegación de Bolivia a los trabajos del Primer Congreso Indigenista Interamericano de Pátzcuaro" y "Partes fundamentales del reglamento de las escuelas indígenas de la República de Bolivia”, III, 1940, vol. III, docs. 1 y 2. 
caso o supuesto triunfo: la creación en 1940 de una institución interamericana específicamente dedicada a la cuestión indígena proporcionó un nuevo espacio de debate y experimentación, que esta vez se pretendía abarcara a todo el continente.

Recibido el 5 de julio de 2010

Aceptado el 22 de septiembre de 2010

\section{Bibliografía}

Gonzalo Aguirre Beltrán: Teoría y práctica de la educación indígena, México, SEP, 1973.

Max A. Bairon: "La educación del indio en Bolivia", América Indígena, II-3, México, 1942, pp. 7-10.

Marten Brienen: "Por qué Warisata no es lo que parece: la escuela-ayllu y el establecimiento del control estatal en la educación indígena", en Nicholas A. Robins (ed.): Cambio y continuidad en Bolivia: etnicidad, cultura e identidad, La Paz, Plural Editores, 2005, pp. 133-150.

Karen Claure: Las Escuelas indígenas como otra forma de resistencia comunitaria, La Paz, Ed. Hisbol, 1989.

Roberto Choque, Vitaliano Soria et al (eds.): Educación indígena: ¿Ciudadanía o colonización?, La Paz, Aruwiyiri, 1992.

Alexander Dawson: "From Model for the Nation to Model Citizen: Indigenismo and the Reivindication of Mexican Indian, 1920-1940", Journal of Latin American Studies, 30-2, Londres, 1998, pp. 279-308.

- "Wild Indian, Mexican Gentlemen and the Lesson Learned in the Casa del Estudiante Indígena, 1926-1932”, The Americas, 57-3, Philadelphia, 2001, pp. 329-361.

- Indian and Nation in Revolutionary Mexico, Tucson, The University of Arizona Press, 2004.

Eve-Marie Fell: "Warisata y la irradiación del núcleo escolar campesino en los Andes (1930-1960)", en Pilar Gonzalbo Aizpuru (coord.): Educación rural e indígena en Iberoamérica, México, El Colegio de México, Universidad Nacional de Educación a Distancia, 1996, pp. 209-223.

Laura Giraudo: "Un maestro popoluca en el Sur de Veracruz: Juan F. González, la Casa del Estudiante Indígena y la educación rural (1924-1931)", Ulúa. Revista de Historia, Sociedad y Cultura, V-10, Xalapa, 2007, pp. 99-138.

- Anular las distancias. Los gobiernos posrevolucionarios en México y la transformación cultural de indios y campesinos, Madrid, Centro de Estudios Políticos y Constitucionales, 2008. 
Antonio Gutiérrez y Oliveros: Valores espirituales de la raza indígena, México, s.e., 1929.

Cecilia Greaves: "La política y el proyecto de educación indígena del avilacamachismo", en María Bertely Busquets (coord.): Historias, saberes indígenas y nuevas etnicidades en la escuela, México, CIESAS, 2006, pp. 95-119.

Instituto Indigenista Interamericano (III): Primer Congreso Indigenista Interamericano, México, III, 1940, 5 vols.

— "Editorial", América Indígena, I-1, México, 1941, pp. 5-6.

Marta Irurozqui: "La ciudadanía clandestina. Democracia y educación indígena en Bolivia, 1826-1952”, Estudios Interdisciplinarios de América Latina y el Caribe, 10-1, Tel Aviv, 1999, pp. 61-87.

Stephen E. Lewis: The Ambivalent Revolution: Forging State and Nation in Chiapas, 1910-1945, Albuquerque, University of New Mexico Press, 2005.

Engracia Loyo: "La empresa redentora. La Casa del Estudiante Indígena", Historia Mexicana, 46-1, México, 1996a, pp. 99-131.

- "Los centros de educación indígena y su papel en el medio rural (19301940)", en Pilar Gonzalbo Aizpuru (coord.): Educación rural e indígena en Iberoamérica, México, El Colegio de México, UNED, 1996b, pp. 139-159.

- Gobiernos revolucionarios y educación popular en México, 1911-1928, México, El Colegio de México, 1999, pp. 292-301.

— "El conocimiento del indio. Nuevo camino para su asimilación", en María Bertely Busquets (coord.): Historias, saberes indígenas y nuevas etnicidades en la escuela, México, CIESAS, 2006, pp. 69-94.

— "El Primer Congreso Indigenista de Pátzcuaro (1940): ¿Génesis de una nueva fórmula de integración del indio americano?", en Manuel Chust e Ivana Frasquet (eds.): Actas XIV Congreso Internacional AHILA. Europa-América, paralelismos en la distancia, Castellón, Universitat Jaume I, Fundación MAPFRE, AHILA, 2008 (edición en CD).

Françoise Martinez: “La création des 'escuelas ambulantes' en Bolivie (1905): instruction, éducation, ou déculturation des masses indigènes?”, en Augustin Redondo (dir.): Relations entre identités culturelles dans l'espace ibérique et ibéro-américain. II: élites et masses, Cahiers de l'UFR d'Etudes Ibériques et Latino-Américaines n¹1, Paris, Presses de la Sorbonne Nouvelle, 1997, pp. 161-171.

— "La peur blanche: un moteur de la politique éducative libérale (1899-1920)", Bulletin de l'Institut Français d'Études Andines, Lima, 1998, 27-2, pp. 265283.

— "Vivre ensemble. Le rôle de l'école libérale dans la construction d'une citoyenneté du XXe siècle", Lazos. Bulletin de liaison bolivianiste, CRIIA, 7, Nanterre, 2005, pp. 29-42.

- «Régénérer la race». Politique éducative en Bolivie (1898-1920), Paris, Éditions de l'IHEAL, 2010. 
Elizardo Pérez: Warisata. La escuela-ayllu, La Paz, Burillo, 1962.

— "Warisata y la Escuela de raíz aborigen", Educación. Revista de pedagogía y orientación sindical (Número especial. Congreso Indigenista de Pátzcuaro), México, 1940.

Luz Olivia Pineda: Caciques culturales. El caso de los maestros bilingües en los altos de Chiapas, Puebla, Altres Costa-Amic, 1993.

Rafael A. Reyeros: Caquiaviri. Escuelas para los Indígenas Bolivianos, La Paz, Ed. Universo, 1946 (1 ed. 1937).

Elsie Rockwell: Hacer escuela, hacer estado: la educación posrevolucionaria vista desde Tlaxcala, México, El Colegio de Michoacán, CIESAS, CINVESTAN, 2007.

Ramón Rubín: La bruma lo vuelve azul, México, FCE, 1984 (1 ed. 1954).

Secretaría de Educación Pública (SEP): La Casa del Estudiante Indígena. 16 meses de labor en un experimento psicológico colectivo con Indios, febrero de 1926-junio de 1927, México, Talleres Gráficos de la Nación, 1927.

- Memoria que indica el estado que guarda el ramo de educación pública el 31 de agosto de 1929, presentada por el Lic. Ezequiel Padilla, México, Talleres Gráficos de la Nación, 1929.

- Memoria que indica el estado que guarda el ramo de educación pública el 31 de agosto de 1931, presentada por el Dr. José Manuel Puig Casauranc, México, Talleres Gráficos de la Nación, 1931.

- Memoria relativa al estado que guarda el ramo de educación pública el 31 de agosto de 1932, México, Talleres Gráficos de la Nación, 1932.

- Memoria relativa al estado que guarda el ramo de educación pública el 31 de agosto de 1933, México, Talleres Gráficos de la Nación, 1933.

- Memoria de la Secretaría de Educación Pública de septiembre de 1936 a agosto de 1937, México, D.A.P.P., 1937.

- México a través de los informes presidenciales, tomo 11: La educación pública, México, Secretaría de la Presidencia, 1976.

Mary Kay Vaughan: "Cultural Approaches to Peasant Politics in the Mexican Revolution”, Hispanic American Historical Review, 79-1, Durham, 1999, pp. 269-305.

- La política cultural en la revolución. Maestros, campesinos y escuelas en México, 1930-1940, México, SEP, FCE, 2000.

Mary Kay Vaughan y Stephen E. Lewis (eds.): The Eagle and the Virgin: Nation and Cultural Revolution in Mexico, 1920-1940, Durham, Duke University Press, 2006.

Adolfo Velasco: La escuela indigenal de Warisata, México, Primer Congreso Indigenista Interamericano, Departamento de Asuntos Indígenas, 1940. 